J. math. fluid mech. 7 (2005) S141-S163

1422-6928/05/02000S141-S23

(C) 2005 Birkhäuser Verlag, Basel

DOI 10.1007/s00021-005-0151-0

Journal of Mathematical

Fluid Mechanics

\title{
The Role of Abstract Numerical Properties in the Change of Character of Reactive Flows
}

\author{
A. Araújo, J. A. Ferreira and P. de Oliveira
}

\begin{abstract}
The aim of this paper is to study the role of explicitness, implicitness and order in the stability and qualitative properties of splitting methods for solving advection-reaction equations. Numerical pathologies produced by simulations are identified which allow the correction of wrong numerical reactive flows. Several numerical examples which show the effectiveness of our approach are presented.
\end{abstract}

Mathematics Subject Classification (2000). 65M06, 65M20, 65M15.

Keywords. Splitting methods, modified equations, qualitative behaviour.

\section{Introduction}

The behaviour of reactive flows can be described by Partial Differential Equations (PDE's) of advection-diffusion-reaction type. These elementary phenomena (advection, diffusion and reaction) are represented by operators that have different properties and the use of tailored numerical methods to construct an approximation for each one of them can be very attractive. For instance if the chemistry is very stiff an implicit approach is required for the reaction part; if the chemistry is non stiff or mildly stiff an explicit approach can lead to enough accuracy. As far as the advection part is concerned explicit approaches are more suitable if non-linear methods like flux-limiter are used; but if advection is discretized in a linear way implicitness could be used.

In the literature there are essentially two ways of constructing tailored methods adapted to the different elementary phenomena described by the PDE: the use of Implicit-Explicit Methods (IMEX) ([2]), and the use of functional splitting ([5], [6], [7], [9], [11]). IMEX methods integrate in time the ODE's system resulting from the semi-discretization of the initial PDE: some of the semi-discretized operators being treated implicitly and others explicitly. Douglas splitting ([3], [6]) and the trapezoidal splitting ([5]) can be viewed as IMEX predictor-corrector methods.

This work has been supported by Centro de Matemática da Universidade de Coimbra and Project POCTI/35039/MAT/2000. 
Functional splitting ([9], [10], [11]) consists in separating the different physical processes by decomposing the initial PDE into several elementary PDE's that describe each one of the flow elementary phenomena. These PDE's can be integrated using different numerical methods and the resulting global numerical approach represents a patching of such tailored methods. Functional splitting methods have better stability than IMEX methods as pointed out in [7]. By the contrary IMEX methods can have better accuracy than functional splitting methods.

In this paper we essentially focuss in functional splitting methods for advectionreaction PDE's. Two main questions are addressed: the dependence of qualitative behaviour and stability of the global method on implicitness, explicitness and order of each one of the tailored methods used in the discretization of the elementary PDE's. Numerical pathologies produced by the simulations are identified, which allow the correction of "wrong" numerical reactive flows. In Section 2 the family of methods is presented. In Section 3 the numerical stability of the splitting methods is studied. In Section 3 the accuracy of some splitting methods is analyzed. In Section 5 a modified PDE, with an infinite number of terms, which exact solution is the numerical solution at the mesh nodes, is constructed. Several numerical examples which show the effectiveness of our approach are presented.

\section{A class of numerical splitting methods}

Let us consider advection-reaction problems of type

$$
\left\{\begin{array}{l}
\frac{\partial u}{\partial t}(x, t)=-\alpha \frac{\partial u}{\partial x}(x, t)+f(x, t, u), x \in \mathbb{R}, t>0, \\
u(x, 0)=u_{0}(x), x \in \mathbb{R},
\end{array}\right.
$$

where $u$ denotes a specie's concentration, $f(x, t, u)$ represents the reaction term and $u_{0}$ stands for the initial concentration.

Let us define in $[0, T]$ the splitting grid $\left\{t_{s}\right\}$ with $t_{s}=s \Delta t$ and $t_{s+1 / 2}=$ $t_{s}+\Delta t / 2$, where $\Delta t$ represents the splitting step size. We suppose that the concentration $u$ at $t=t_{s}$ is known (at least approximately). The computation of an approximation of $u$ at time level $t_{s+1}$ is obtained considering a splitting algorithm based on the decomposition of problem (1) in three subproblems - respectively, an advection problem in $\left[t_{s}, t_{s+1 / 2}\right]$, a reaction problem in $\left[t_{s}, t_{s+1}\right]$ and a final advection problem in $\left[t_{s+1 / 2}, t_{s+1}\right]$ - linked as follows:

$$
\begin{gathered}
\frac{\partial v}{\partial t}(x, t)=-\alpha \frac{\partial v}{\partial x}, t \in\left[t_{s}, t_{s+1 / 2}\right], \quad v\left(t_{s}\right)=u\left(t_{s}\right), \\
\frac{d w}{d t}=f(x, t, w), t \in\left[t_{s}, t_{s+1}\right], \quad w\left(t_{s}\right)=v\left(t_{s+1 / 2}\right), \\
\frac{\partial p}{\partial t}(x, t)=-\alpha \frac{\partial p}{\partial x}, t \in\left[t_{s+1 / 2}, t_{s+1}\right], \quad p\left(t_{s+1 / 2}\right)=w\left(t_{s+1}\right) .
\end{gathered}
$$


The splitting solution $p\left(t_{s+1}\right)$ at $t=t_{s+1}$ - which is the exact solution of (2)-(4) - represents an approximation of $u\left(t_{s+1}\right)$.

For a reaction term of type $f(u)$ not depending on $x$ and $t$ we can easily establish that $p\left(t_{s+1}\right)=u\left(t_{s+1}\right)([1])$ that is there is no splitting error. In the case where $f$ depends on $x, t$ and $u$ we have

$$
\begin{gathered}
u(x, t)=\int_{0}^{t} f(\alpha \tau+x-\alpha \Delta t, \tau, u(\alpha \tau+x-\alpha \Delta t, \tau)) d \tau+u_{0}(x-\alpha \Delta t), \\
p(x, t)=\int_{0}^{t} f\left(x-\frac{\alpha \Delta t}{2}, \tau, w\left(x-\frac{\alpha \Delta t}{2}, \tau\right)\right) d \tau+u_{0}(x-\alpha \Delta t),
\end{gathered}
$$

and assuming that the partial derivatives of $f$ are bounded it can be easily established that

$$
\|u-p\|_{\infty}=\mathcal{O}(\Delta t)
$$

If an initial-boundary value problem of type

$$
\left\{\begin{array}{l}
\frac{\partial u}{\partial t}(x, t)=-\alpha \frac{\partial u}{\partial x}+f(x, t, u), x \in \mathbb{R}^{+}, t>0 \\
u(x, 0)=u_{0}(x), x \in \mathbb{R}^{+} \\
u(0, t)=u_{1}(t), t \geq 0
\end{array}\right.
$$

is considered, it has been established in [1] that (5) holds. This estimation can be improved as mentioned in [7].

We consider in what follows several numerical schemes for the computation of a numerical approximation of the splitting solution $p$ in a space domain $[a, b]$. These schemes have been obtained by patching together methods with different implicitness, explicitness and order properties.

Let us define the grid $\left\{x_{j}\right\}$ in the space domain $[a, b]$ with $x_{j}-x_{j-1}=h=\frac{b-a}{n}$, for $j=1, \ldots, n, x_{0}=a$. Let $v_{h}^{s}, w_{h}^{s}$ and $p_{h}^{s}$ be numerical approximations at $t_{s}$ of $v\left(t_{s}\right), w\left(t_{s}\right)$ and $p\left(t_{s}\right)$ respectively. We discretize (2), (3) and (4) respectively by

$$
\begin{gathered}
v_{h}^{s+1 / 2}=A_{h} v_{h}^{s}, \quad v_{h}^{s}=u_{h}^{s}, \\
F_{1, h}\left(w_{h}^{s+1}\right)=F_{2, h}\left(w_{h}^{s}\right), w_{h}^{s}=v_{h}^{s+1 / 2}, \\
p_{h}^{s+1}=A_{h} p_{h}^{s+1 / 2}, \quad p_{h}^{s+1 / 2}=w_{h}^{s+1},
\end{gathered}
$$

where $v_{h}^{s+1 / 2}$ represents a numerical approximation of $v$ at $t=t_{s}+\frac{\Delta t}{2}, w_{h}^{s+1}$ represents a numerical approximation of $w$ at $t=t_{s+1}$ and $p_{h}^{s+1}$ represents a numerical approximation of the splitting solution $p$ at $t_{s+1}$ and consequently of $u\left(t_{s+1}\right)$. In the algorithm (6)-(8), $A_{h}$ is a linear operator resulting from the discretization of the advection equation (2) and it is assumed that the discretization of (3) can be rewritten as in (7). The operators $A_{h}, F_{i, h}, i=1,2$, take into account the boundary conditions prescribed for (2)-(4). As an example let us consider in (6) and (8) 
first order explicit methods $\left(E_{1}^{a}\right)$ and, for a reaction term of type $f(u)$ in (7), a first order implicit method $\left(I_{1}^{r}\right)$. We have

$$
\begin{gathered}
\frac{v_{j}^{s+1 / 2}-v_{j}^{s}}{\Delta t / 2}+\alpha \frac{v_{j}^{s}-v_{j-1}^{s}}{h}=0, v_{j}^{s}=u_{j}^{s}, \\
\frac{w_{j}^{s+1}-w_{j}^{s}}{\Delta t}=f\left(w_{j}^{s+1}\right), w_{j}^{s}=v_{j}^{s+1 / 2}, \\
\frac{p_{j}^{s+1}-p_{j}^{s+1 / 2}}{\Delta t / 2}+\alpha \frac{p_{j}^{s+1 / 2}-p_{j-1}^{s+1 / 2}}{h}=0, p_{j}^{s+1 / 2}=w_{j}^{s+1} .
\end{gathered}
$$

In this case the matrix $A_{h}$ has non null entries in the principal and lower diagonals respectively $1-\frac{\alpha \Delta t}{2 h}$ and $\frac{\alpha \Delta t}{2 h} ; F_{1, h}$ and $F_{2, h}$ are defined by $F_{1, h}=I-\frac{\Delta t}{2} f$ and $F_{2, h}=I$ respectively. The global method arising from (9)-(11) is then

$$
u_{h}^{s+1}=A_{h}^{2} u_{h}^{s}+A_{h} \Delta t f\left(A_{h}^{-1} u_{h}^{s+1}\right),
$$

with $f\left(A_{h}^{-1} u_{h}^{s+1}\right)=\left(f\left(\left(A_{h}^{-1} u_{h}^{s+1}\right)_{i}\right)\right)$.

There are several possible couplings of methods. To construct these methods, we introduce the following notations $\left(f\left(w_{j}^{s}\right)\right.$ represents $\left.f\left(x_{j}, t_{s}, w_{j}^{s}\right)\right)$ :

- $E_{1}^{a}$ - First order explicit for advection

$$
\frac{v_{j}^{s+1}-v_{j}^{s}}{\Delta t}+\alpha \frac{v_{j}^{s}-v_{j-1}^{s}}{h}=0 ;
$$

- $I_{1}^{a}$ - First order implicit for advection

$$
\frac{v_{j}^{s+1}-v_{j}^{s}}{\Delta t}+\alpha \frac{v_{j}^{s+1}-v_{j-1}^{s+1}}{h}=0
$$

- $E_{1}^{r}$ - First order explicit for reaction

$$
\frac{w_{j}^{s+1}-w_{j}^{s}}{\Delta t}=f\left(w_{j}^{s}\right)
$$

- $E_{2}^{r}$ - Second order explicit for reaction (Runge-Kutta method)

$$
\frac{w_{j}^{s+1}-w_{j}^{s}}{\Delta t}=(1-\gamma) f\left(w_{j}^{s}\right)+\gamma f\left(w_{j}^{s}+\frac{\Delta t}{2 \gamma} f\left(w_{j}^{s}\right)\right) ;
$$

- $I_{1}^{r}$ - First order implicit for reaction

$$
\frac{w_{j}^{s+1}-w_{j}^{s}}{\Delta t}=f\left(w_{j}^{s+1}\right)
$$

- $I_{2}^{r}$ - Second order implicit for reaction

$$
\frac{w_{j}^{s+1}-w_{j}^{s}}{\Delta t}=\frac{1}{2}\left(f\left(w_{j}^{s+1}\right)+f\left(w_{j}^{s}\right)\right) \text {. }
$$


From (12)-(17) we obtain the splitting methods that are presented, in its final forms, in Table 1 . In this table, for example, method $I_{1}^{a} E_{1}^{r} I_{1}^{a}$ is a method resulting from the use of $I_{1}^{a}$ to integrate (2) and (4) and $E_{1}^{r}$ to integrate (3). These forms will be used in Section 3, 4 and 5 to study, respectively, stability properties, accuracy and qualitative properties.

\begin{tabular}{|l||l|}
\hline Method & Formula \\
\hline \hline$E_{1}^{a} E_{1}^{r} E_{1}^{a}$ & $u_{h}^{s+1}=A_{h}^{2} u_{h}^{s}+\Delta t A_{h} f\left(A_{h} u_{h}^{s}\right)$ \\
$E_{1}^{a} E_{2}^{r} E_{1}^{a}$ & $u_{h}^{s+1}=A_{h}^{2} u_{h}^{s}+(1-\gamma) \Delta t A_{h} f\left(A_{h}^{-1} u_{h}^{s+1}\right)+\gamma \Delta t A_{h} f\left(A_{h} u_{h}^{s}\right)$ \\
$E_{1}^{a} I_{1}^{r} E_{1}^{a}$ & $u_{h}^{s+1}=A_{h}^{2} u_{h}^{s}+\Delta t A_{h} f\left(A_{h}^{-1} u_{h}^{s+1}\right)$ \\
$E_{1}^{a} I_{2}^{r} E_{1}^{a}$ & $u_{h}^{s+1}=A_{h}^{2} u_{h}^{s}+\frac{\Delta t}{2}\left(A_{h} f\left(A_{h}^{-1} u_{h}^{s+1}\right)+A_{h} f\left(A_{h} u_{h}^{s}\right)\right)$ \\
$I_{1}^{a} E_{1}^{r} I_{1}^{a}$ & $B_{h}^{2} u_{h}^{s+1}=u_{h}^{s}+\Delta t B_{h} f\left(B_{h}^{-1} u_{h}^{s}\right)$ \\
$I_{1}^{a} E_{2}^{r} I_{1}^{a}$ & $B_{h}^{2} u_{h}^{s+1}=u_{h}^{s}+\Delta t(1-\gamma) B_{h} f\left(B_{h} u_{h}^{s}\right)+\Delta t \gamma B_{h} f\left(f\left(B_{h}^{-1} u_{h}^{s}\right)+\frac{\Delta t}{2 \gamma} f\left(B_{h}^{-1} u_{h}^{s}\right)\right)$ \\
$I_{1}^{a} I_{1}^{r} I_{1}^{a}$ & $B_{h}^{2} u_{h}^{s+1}=u_{h}^{s}+\Delta t B_{h} f\left(B_{h} u_{h}^{s+1}\right)$ \\
$I_{1}^{a} I_{2}^{r} I_{1}^{a}$ & $B_{h}^{2} u_{h}^{s+1}=u_{h}^{s}+\frac{\Delta t}{2}\left(B_{h} f\left(B_{h} u_{h}^{s+1}\right)+B_{h} f\left(B_{h}^{-1} u_{h}^{s}\right)\right)$ \\
\hline
\end{tabular}

TABLE 1. Numerical methods.

In this table $B_{h}$ denotes a matrix with non null entries in the principal and lower diagonal represented by respectively $-\frac{\alpha \Delta}{2 h}$ and $1+\frac{\alpha \Delta}{2 h}$.

In Section 4 we also consider the explicit and implicit non splitting methods NSE, NSI - defined respectively by

and

$$
\frac{u_{j}^{s+1}-u_{j}^{s}}{\Delta t}+\alpha \frac{u_{j}^{s}-u_{j-1}^{s}}{h}=f\left(u_{j}^{s}\right)
$$

$$
\frac{u_{j}^{s+1}-u_{j}^{s}}{\Delta t}+\alpha \frac{u_{j}^{s+1}-u_{j-1}^{s+1}}{h}=f\left(u_{j}^{s+1}\right)
$$

\section{Numerical stability of splitting methods}

In this section we study the numerical stability of the class of splitting methods presented in Section 2.

Let $\tilde{v}_{h}^{s}, \tilde{w}_{h}^{s}$ and $\tilde{p}_{h}^{s}$ be perturbed numerical solutions of (6), (7) and (8), respectively. With $u_{h}^{s+1}=p_{h}^{s+1}$ and $\tilde{u}_{h}^{s+1}=\tilde{p}_{h}^{s+1}$ we have

$$
u_{h}^{s+1}-\tilde{u}_{h}^{s+1}=A_{h} J F_{1, h}^{-1} J F_{2, h} A_{h}\left(u_{h}^{s}-\tilde{u}_{h}^{s}\right)
$$

where

$$
J F_{1, h}=\left[\frac{\partial F_{1, h}^{(i)}}{\partial y_{j}}\left(\theta_{j}^{s+1}\right)\right], J F_{2, h}=\left[\frac{\partial F_{2, h}^{(i)}}{\partial y_{j}}\left(\theta_{j}^{s}\right)\right]
$$


and

$$
\theta_{j}^{\ell}=\left(\tilde{w}_{1}^{\ell}, \ldots, \tilde{w}_{j-1}^{\ell}, \theta \tilde{w}_{j}^{\ell}+(1-\theta) w_{j}^{\ell}, w_{j+1}^{\ell}, \ldots, w_{n-1}^{\ell}\right), \theta \in(0,1) .
$$

From (18) we have

$$
\left\|u_{h}^{s+1}-\tilde{u}_{h}^{s+1}\right\|_{\infty} \leq\left\|A_{h}\right\|_{\infty}^{2}\left\|J F_{1, h}^{-1}\right\|_{\infty}\left\|J F_{2, h}\right\|_{\infty}\left\|u_{h}^{s}-\tilde{u}_{h}^{s}\right\|_{\infty} .
$$

Let us represent by $S(r, \Delta t, f)$ with $r=\frac{\alpha \Delta t}{2 h}$ the stability coefficient

$$
\left\|A_{h}\right\|_{\infty}^{2}\left\|J F_{1, h}^{-1}\right\|_{\infty}\left\|J F_{2, h}\right\|_{\infty}
$$

To study the numerical stability properties of the class, $S(r, \Delta t, f)$ is computed for some of the methods considered before. We represent by $D$ the domain $[0,1] \times[0, T] \times\left[\min _{(x, t)} u-\rho, \max _{(x, t)} u+\rho\right]$ where $\rho$ is a positive constant. Let $B_{\rho}\left(u\left(t_{s}\right)\right)$ be the open ball with center $u\left(t_{s}\right)$ and radius $\rho$. By $f^{\prime}$ we denote the partial derivative of $f$ with respect to the third argument.

Proposition 1. Let $u_{h}^{s+1}$ and $\tilde{u}_{h}^{s+1}$ be two numerical approximations of $u\left(t_{s+1}\right)$ computed from approximations $u_{h}^{s}, \tilde{u}_{h}^{s} \in B_{\rho}\left(u\left(t_{s}\right)\right)$. Then the following estimates hold:

1. For $I_{1}^{a} E_{1}^{r} I_{1}^{a}$ we have

$$
\left\|u_{h}^{s+1}-\tilde{u}_{h}^{s+1}\right\|_{\infty} \leq \max _{(x, t) \in(x, t, y) \in D}\left|1+\Delta t f^{\prime}\right|\left(1-\left(\frac{r}{1+r}\right)^{1 / h}\right)^{2}\left\|u_{h}^{s}-\tilde{u}_{h}^{s}\right\|_{\infty}
$$

2. For $E_{1}^{a} I_{1}^{r} E_{1}^{a}$ we have

$$
\left\|u_{h}^{s+1}-\tilde{u}_{h}^{s+1}\right\|_{\infty} \leq \max _{(x, t, y) \in D} \frac{1}{\left|1-\Delta t f^{\prime}\right|}\left((1-r)^{2}+r^{2}+2 r|1-r|\right)\left\|u_{h}^{s}-\tilde{u}_{h}^{s}\right\|_{\infty}
$$

3. For $I_{1}^{a} E_{2}^{r} I_{1}^{a}$ we have

$$
\begin{gathered}
\left\|u_{h}^{s+1}-\tilde{u}_{h}^{s+1}\right\|_{\infty} \leq\left(\max _{(x, t, y) D}\left(1+\frac{\Delta t^{2}}{2} f^{\prime 2}\right)+\max _{(x, t, y) \in D}\left|1+\Delta t f^{\prime}\right|\right) \\
\left(1-\left(\frac{r}{1+r}\right)^{1 / h}\right)^{2}\left\|u_{h}^{s}-\tilde{u}_{h}^{s}\right\|_{\infty} .
\end{gathered}
$$

We note that two factors can be identified in the stability coefficient $S(r, \Delta t, f)$ : the factor $\left\|J F_{1, h}^{-1}\right\|_{\infty}\left\|J F_{2, h}\right\|_{\infty}$ arising from the discretization of the reaction equation (3) and the term $\left\|A_{h}\right\|_{\infty}^{2}$ corresponding to the discretization of advection equations (2) and (4). These two factors balance, in some sense, each other. In

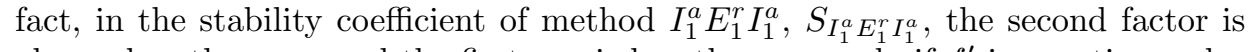
always less than one and the first one is less than one only if $f^{\prime}$ is negative and

$$
\Delta t \leq \frac{2}{\max \left(-f^{\prime}\right)}
$$


This restriction can be relaxed, while guaranteing stability, provided that the stability coefficient, $S_{I_{1}^{a} E_{1}^{r} I_{1}^{a}}$, is such that

$$
S_{I_{1}^{a} E_{1}^{r} I_{1}^{2}} \leq 1 .
$$

For method $E_{1}^{a} I_{1}^{r} E_{1}^{a}$, the factor $\frac{1}{\left|1-\Delta t f^{\prime}\right|}$ is less than one if $f^{\prime}<0$ and the second factor is larger than one unless $r<1$. Again this last restriction can be relaxed provided that the stability factor satisfies

$$
S_{E_{1}^{a} I_{1}^{r} E_{1}^{a} \leq 1}
$$

We remark that the stability coefficients of $I_{1}^{a} E_{1}^{r} I_{1}^{a}$ and $I_{1}^{a} E_{2}^{r} I_{1}^{a}$ have in common a term, $G(r, h)$,

$$
G(r, h):=\left(1-\left(\frac{r}{1+r}\right)^{1 / h}\right)^{2},
$$

arising from the implicit discretization of the two advection equations. $G(r, h)$ is plotted in Figure 1 for different values of $r$.

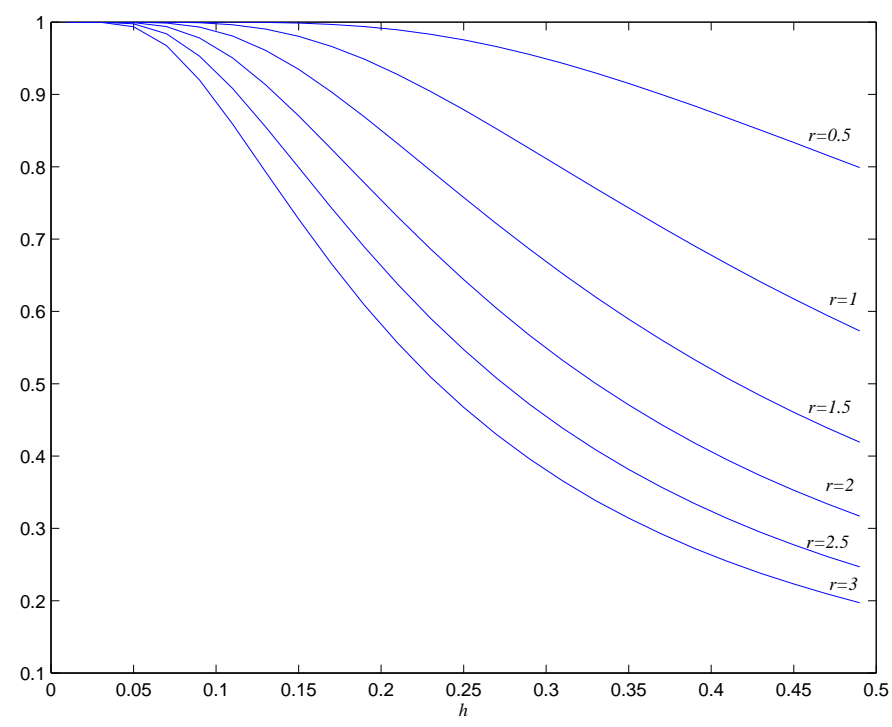

FIG. 1. The factor $G(r, h)$.

If $1>\epsilon>0$ is fixed, then, for $h>0$ and $r$ such that

$$
\frac{(1-\epsilon)^{h}}{1-(1-\epsilon)^{h}} \leq r
$$


$G(r, h) \leq \epsilon^{2}$ and consequently the stability coefficient of both methods can be less than one. In fact, if

$$
\max _{(x, t) \in(x, t, y) \in D}\left|1+\Delta t f^{\prime}\right|<\frac{1}{\epsilon^{2}}
$$

then (23) holds; if

$$
\max _{(x, t, y) D}\left(1+\frac{\Delta t^{2}}{2} f^{\prime 2}\right)+\max _{(x, t, y) \in D}\left|1+\Delta t f^{\prime}\right|<\frac{1}{\epsilon^{2}}
$$

then $S_{I_{1}^{a} E_{2}^{r} I_{1}^{a}} \leq 1$.

In Figure $2-5$ we present several numerical solutions of the initial-boundary value problem

$$
\left\{\begin{array}{l}
\frac{\partial u}{\partial t}(x, t)=-\alpha \frac{\partial u}{\partial x}(x, t)+k u^{2}(x, t), x \in(0,1), t>0 \\
u(x, 0)=1, x \in(0,1) \\
u(0, t)=1 t>0
\end{array}\right.
$$

where $k=-20$, obtained for $t=10$. The aim of these experiments is: to compare the stability properties of NSE, $E_{1}^{a} E_{1}^{r} E_{1}^{a}, I_{1}^{a} E_{1}^{r} I_{1}^{a}$ and $E_{1}^{a} I_{1}^{r} E_{1}^{a}$ when the same Courant Number, $C F L$, is used; to establish a stability route for each of the mentioned methods, when $C F L$ increases.

We note that for $C F L=0.25$ (Figure 2) the methods present an analogous behaviour. The different diffusion properties they exhibit will be explained in Section 5. For $C F L=0.5$ (Figure 3) NSE is unstable and $E_{1}^{a} E_{1}^{r} E_{1}^{a}$ shows some signs of instability. In Figure 4 the numerical experiments have been performed with $C F L=0.75$. The instability of $E_{1}^{a} E_{1}^{r} E_{1}^{a}$ for $C F L=0.5$ is now clearly exhibited. We also observed in $E_{1}^{a} I_{1}^{r} E_{1}^{a}$ some instabilities. Finally in Figure 5 all the methods except $I_{1}^{a} E_{1}^{r} I_{1}^{a}$ present an unstable behaviour. In all numerical experiments $h=0.1$ and $\Delta t=0.05$ have been considered in order that (22) is satisfied.

When the reaction is stiff explicit methods for advection and implicit methods for reaction should be used; for non stiff or mildly stiff reactions explicit methods in reaction and implicit in advection should be preferred. In fact, for a stiff problem, condition (22) can be very severe. The use of implicit methods for reaction eliminates such condition introducing, for example, a condition of type $r<1$, for $E_{1}^{a} I_{1}^{r} E_{1}^{a}$, arising from explicit discretization of advection. For a non stiff or mildly stiff reaction the use of implicit methods in advection and explicit methods in reaction will lead to a less restrictive stability condition. The previous considerations allow us to give a rigorous support to the guidelines generally pointed out in the literature ([7], [10], [11]). 

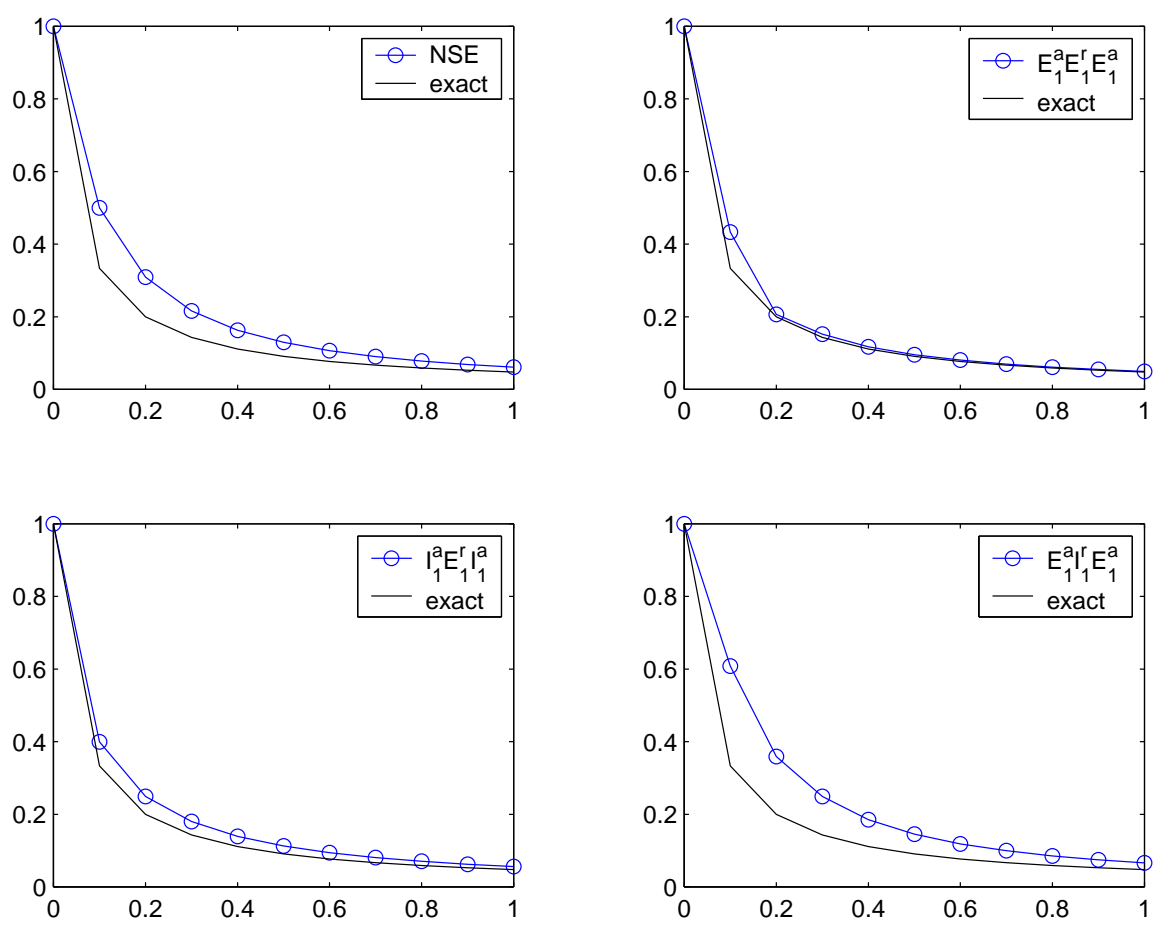

FIG. 2. $\alpha=1, C F L=0.25$.

\section{Accuracy of splitting methods}

Let $u\left(t_{s}\right)$ be the solution of the advection-reaction equation at $t=t_{s}$ and $u_{h}^{s}$ its approximation computed using splitting method (6)-(8). Let $p\left(t_{s}\right)$ be the solution computed using the functional splitting (2), (3) and (4). We have

$$
\left\|u\left(t_{s}\right)-u_{h}^{s}\right\|_{\infty} \leq\left\|u\left(t_{s}\right)-p\left(t_{s}\right)\right\|_{\infty}+\left\|p\left(t_{s}\right)-u_{h}^{s}\right\|_{\infty} .
$$

We estimate in what follows $\left\|p\left(t_{s}\right)-u_{h}^{s}\right\|_{\infty}$.

Let $T_{1, h}^{s+1 / 2}$ be the truncation errors at $t_{s+1 / 2}$ associated with discretizations (6), and $T_{i, h}^{s+1}, i=2,3$, be the truncation errors at $t=t_{s+1}$ associated with discretizations (7) and (8) respectively. By $e_{1, h}^{s+1+1 / 2}$ and $e_{i, h}^{s+1}, i=2,3$, we denote the global errors defined by $e_{1, h}^{s+1 / 2}=v\left(t_{s+1 / 2}\right)-v_{h}^{s+1 / 2}, e_{2, h}^{s+1}=w\left(t_{s+1}\right)-w_{h}^{s+1}$ 

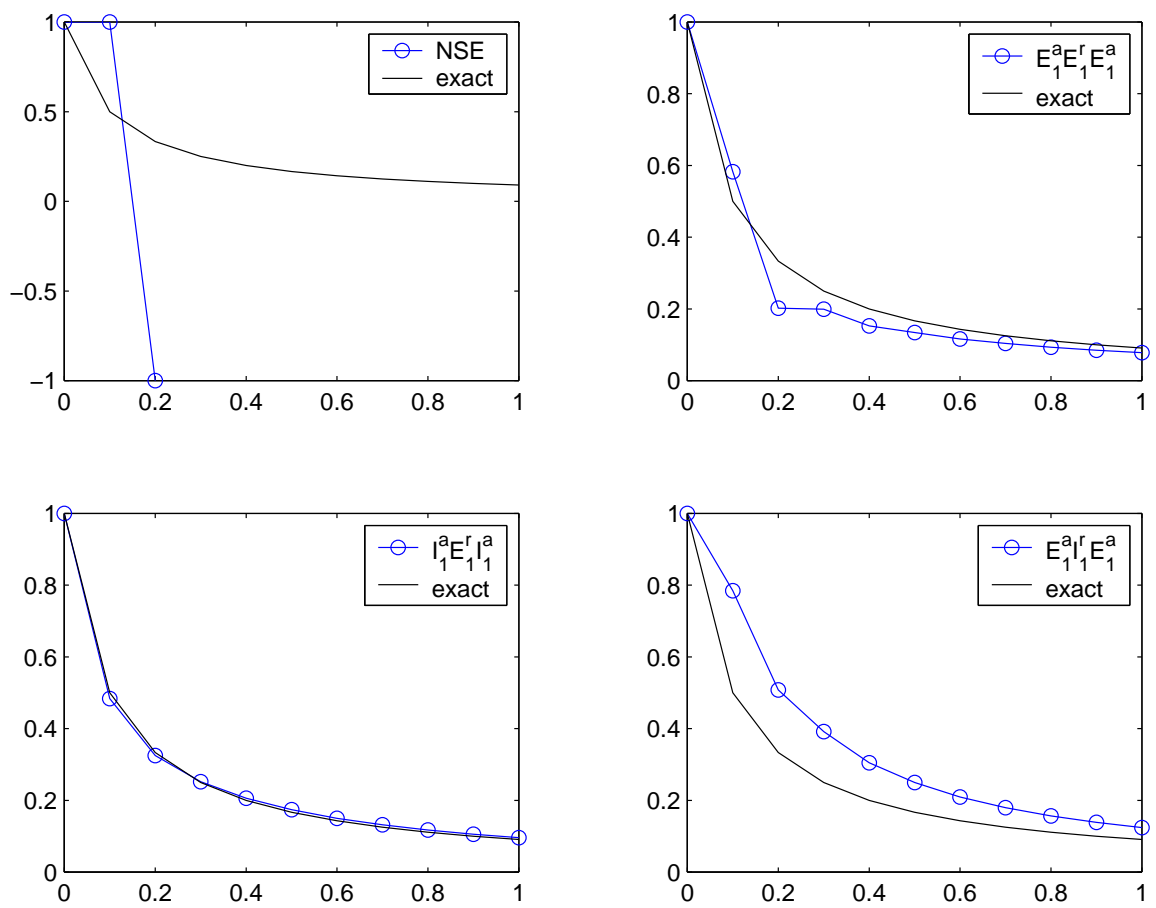

FIG. 3. $\alpha=2, C F L=0.5$.

and $e_{3, h}^{s+1}=p\left(t_{s+1}\right)-p_{h}^{s+1}$. These errors satisfy

$$
\begin{aligned}
\left\|e_{3, h}^{s+1}\right\|_{\infty} \leq & \left\|A_{h}\right\|_{\infty}\left\|e_{2, h}^{s+1}\right\|_{\infty}+\Delta t\left\|T_{3, h}^{s+1}\right\|_{\infty} \\
\leq & \left\|A_{h}\right\|_{\infty}\left\|J F_{1, h}^{-1}\right\|_{\infty}\left(\left\|J F_{2, h}\right\|_{\infty}\left\|e_{1, h}^{s+1 / 2}\right\|_{\infty}+\Delta t\left\|T_{2, h}^{s+1}\right\|_{\infty}\right)+\Delta t\left\|T_{3, h}^{s+1}\right\|^{\infty} \\
\leq & \left\|A_{h}\right\|_{\infty}^{2}\left\|J F_{1, h}^{-1}\right\|_{\infty}\left\|J F_{2, h}\right\|_{\infty}\left\|e_{1, h}^{s}\right\|_{\infty} \\
& +\Delta t\left\|A_{h}\right\|_{\infty}\left(\left\|J F_{1, h}^{-1}\right\|_{\infty}\left\|J F_{2, h}\right\|_{\infty}\left\|T_{1, h}^{s+1 / 2}\right\|_{\infty}+\left\|T_{2, h}^{s+1}\right\|_{\infty}\right)+\Delta t\left\|T_{3, h}^{s+1}\right\|_{\infty} .
\end{aligned}
$$

Therefore, we obtain the following inequality for the splitting discretization error

$$
\left\|p\left(t_{s+1}\right)-u_{h}^{s+1}\right\|_{\infty} \leq S(r, \Delta t, f)\left\|p\left(t_{s}\right)-u_{h}^{s}\right\|_{\infty}+\Delta t\left\|T_{h}^{s+1}\right\|_{\infty},
$$

where

$$
\left\|T_{h}^{s+1}\right\|_{\infty}=\left\|A_{h}\right\|_{\infty}\left(\left\|J F_{1, h}^{-1}\right\|_{\infty}\left\|J F_{2, h}\right\|_{\infty}\left\|T_{1, h}^{s+1 / 2}\right\|_{\infty}+\left\|T_{2, h}^{s+1}\right\|_{\infty}\right)+\left\|T_{3, h}^{s+1}\right\|_{\infty} .
$$

Proposition 2. If the splitting method (6)-(8) has a stability coefficient $S(r, \Delta t, f)$ such that

$$
S(r, \Delta t, f)<1,
$$



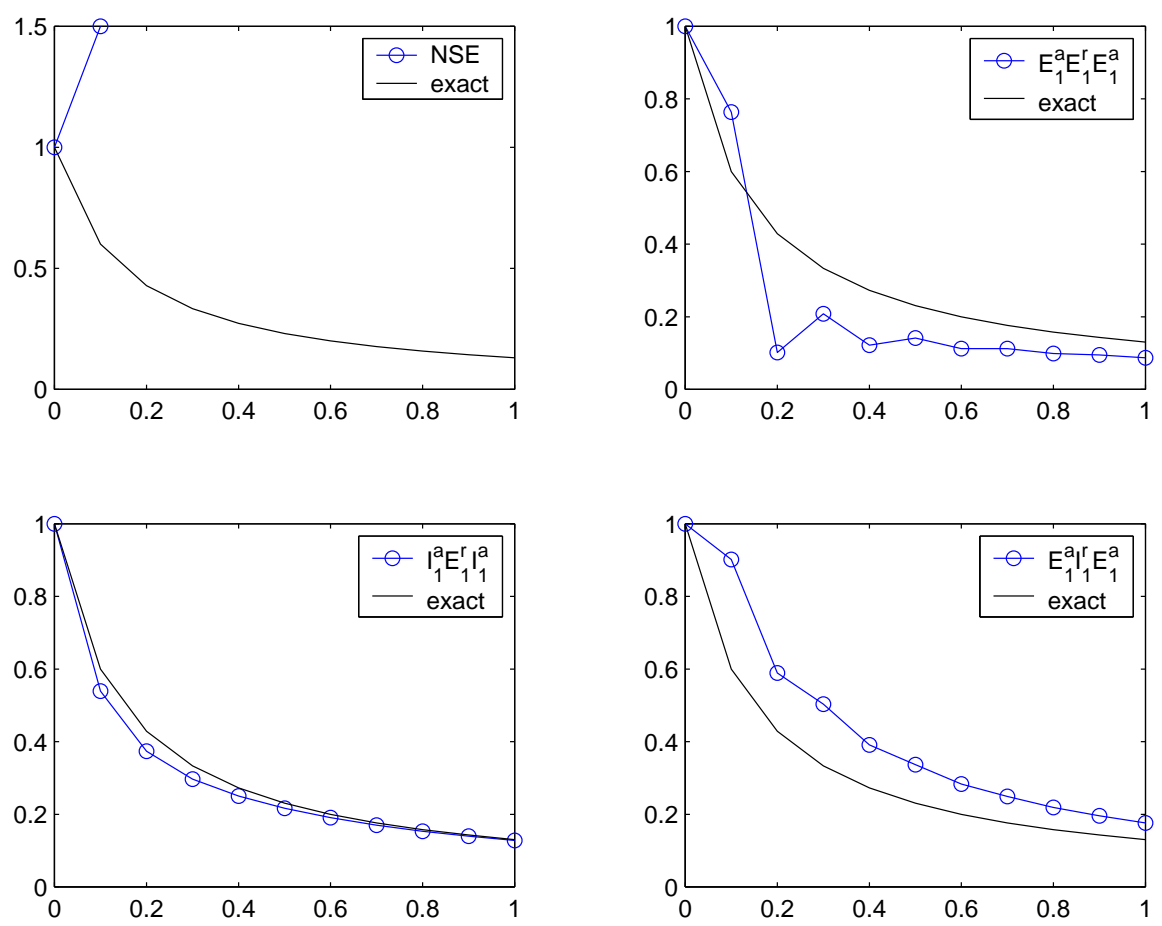

FIG. 4. $\alpha=3, C F L=0.75$.

then

$\left\|p\left(t_{s}\right)-u_{h}^{s}\right\|_{\infty} \leq \frac{1-S(r, \Delta t, f)^{s+1}}{1-S(r, \Delta t, f)} S_{e} \max _{\ell=0, \ldots, s}\left\{\left\|T_{1, h}^{s+1 / 2}\right\|_{\infty},\left\|T_{2, h}^{s+1}\right\|_{\infty},\left\|T_{3, h}^{s+1}\right\|_{\infty}\right\}$,

where $S_{e}$ satisfies

$$
\max \left\{\left\|A_{h}\right\|_{\infty}\left\|J F_{1, h}^{-1}\right\|_{\infty}\left\|J F_{2, h}\right\|_{\infty},\left\|A_{h}\right\|_{\infty}\right\} \leq S_{e}
$$

The accuracy of splitting methods is defined by comparing the numerical splitting solution $u_{h}^{s}$ with the splitting solution $p\left(t_{s}\right)$.

Corollary 1. 1. If $f^{\prime}<0$ and $\Delta t$ satisfies (22), then $I_{1}^{a} E_{1}^{r} I_{1}^{a}$ is first order accurate;

2. If $f^{\prime}<0$ and $r<1$ then $E_{1}^{a} I_{1}^{r} E_{1}^{a}$ is first order accurate. 

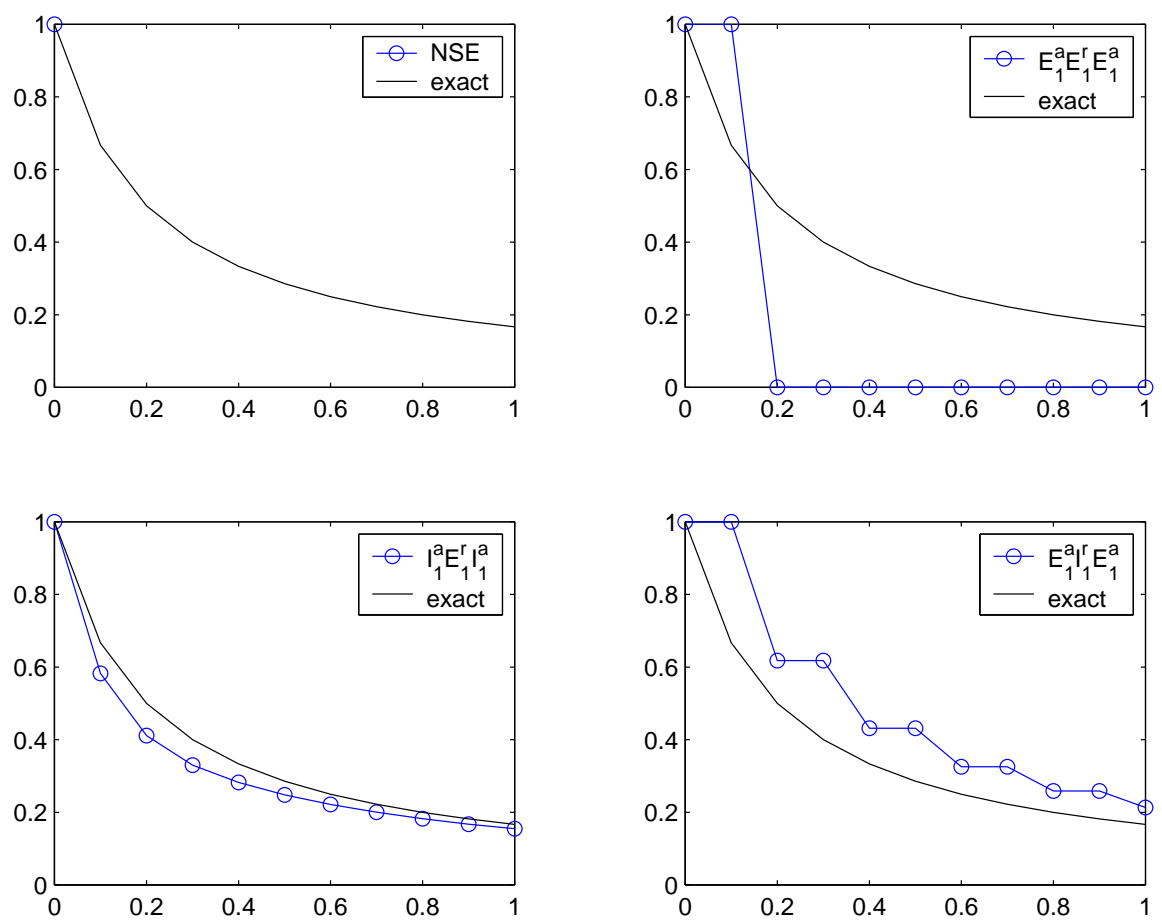

FIG. 5. $\alpha=4, C F L=1$.

\section{Qualitative properties}

Let us consider an advection-diffusion-reaction equation of type

$$
\frac{\partial \bar{u}}{\partial t}=-\alpha \frac{\partial \bar{u}}{\partial x}+\beta \frac{\partial^{2} \bar{u}}{\partial x^{2}}+f(\bar{u}),
$$

where $\alpha, \beta>0$.

For a loss reaction we consider $f(\bar{u})=k \bar{u}^{2}, k \in \mathbb{R}^{-}$. The effect of the variation of parameters $\alpha, \beta$ and $k$ can be observed in Figures 6 and 7. We note that, as diffusion increases the loss decreases, as advection increases the loss also decreases and as reaction increases the loss increases. The plots have been obtained with a standard numerical method in a refined space-time mesh.

In the case of a production reaction described by $f(\bar{u})=k(1-\bar{u})^{2}, k \in \mathbb{R}^{+}$, analogous conclusions can be established (Figure 7).

From Figures 6 and 7 we can conclude that, if two reactive flows differ by only one parameter (advection speed, reaction speed or diffusion coefficient), it is possible to make the correspondence between the flow and this parameter. However, if there are different rates of more than one elementary phenomena, it is sometimes impossible to make the correspondence between parameters and flow. We can 
Vol. 7 (2005)
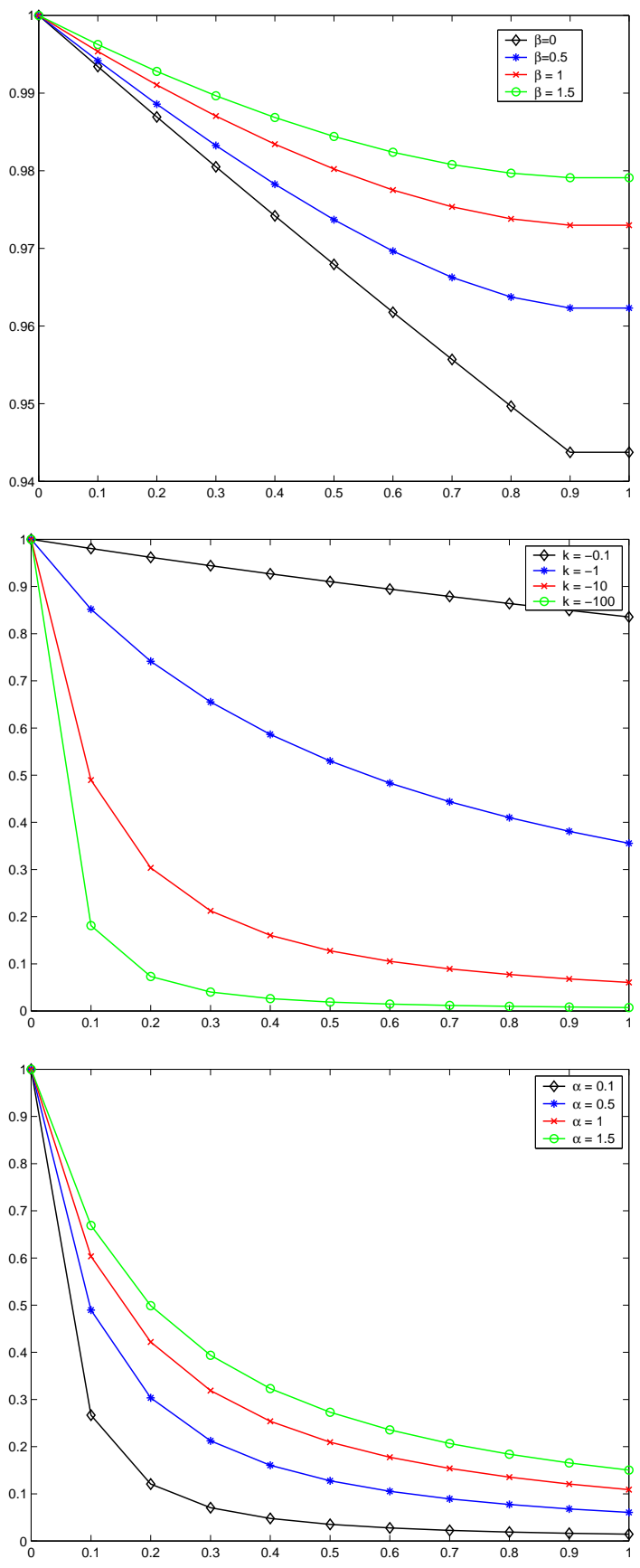

FIG. 6. Effects of elementary phenomena in a loss reaction: (a) diffusion $(\alpha=1.5, k=-0.1)$; (b) reaction $(\alpha=0.5, \beta=0)$ and (c) advection $(\beta=0, k=-10)$. 

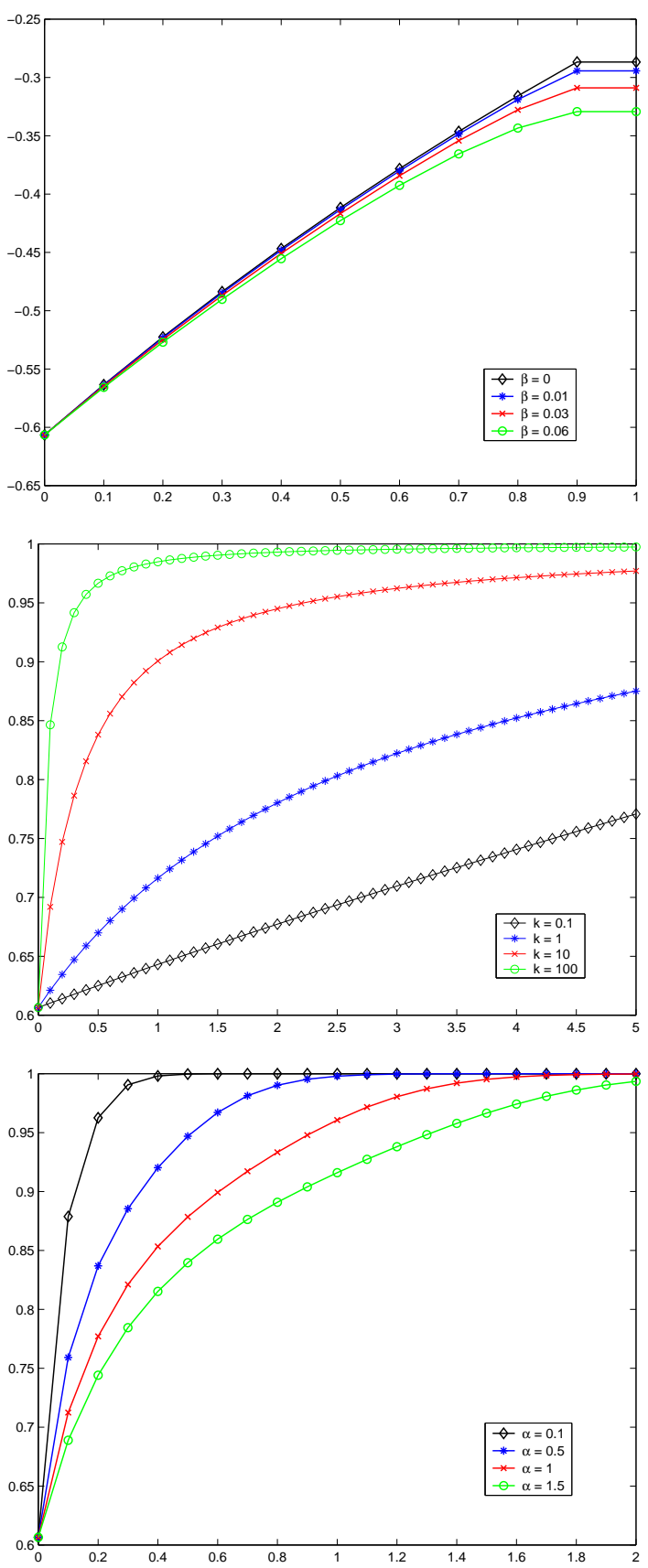

FIG. 7. Effects of elementary phenomena: (a) diffusion $(\alpha=0.5, k=0.1)$; (b) reaction $(\alpha=1.2, \beta=0)$ and (c) advection $(\beta=0, k=10)$. 
observe this fact in Figure 8 where the plots of solutions of two advection-reaction equations with the reaction term $f(\bar{u})=k \bar{u}^{2}, k \in \mathbb{R}^{-}$, differing by both advection coefficient and reaction speed, are exactly the same.

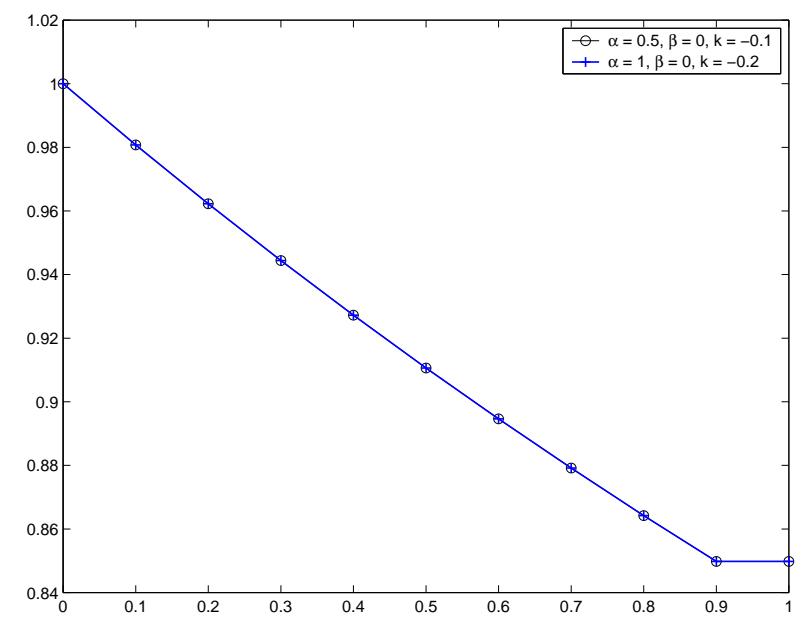

FIG. 8. Effects of elementary phenomena: different rates of more than one phenomena.

The previous considerations will be used in what follows to interpret the "wrong" flows produced by some of the numerical splitting methods presented in Section 2.

Let $F(u)=0$ represent a PDE. Discretizing this equation with one of the splitting methods of Table II, and assuming that $u$ is smooth enough, we can construct, using Taylor series, a modified PDE represented by $F(\bar{u})+E(\bar{u})=0$, where $\bar{u}$ stands for an interpolation function for the numerical solution $u_{h}^{s}$ and $E(\bar{u})$ represents an infinite number of differential terms. In the case of equation (1) with a reaction term $f(\bar{u})$, for a numerical splitting method, a modified equation will take a form of type

$\frac{\partial \bar{u}}{\partial t}=-\alpha \frac{\partial \bar{u}}{\partial x}+f(\bar{u})+C_{1} \Delta t \frac{\partial \bar{u}}{\partial x}+C_{2} F_{1}(\Delta t, h) \frac{\partial^{2} \bar{u}}{\partial x^{2}}+\Delta t C_{3} F_{2}\left(f, f^{\prime}\right)+\mathcal{O}\left(\Delta t^{2}, h^{2}\right)$,

where $C_{i}, i=1,2,3$, represent some real constants. This modified equation has a speed advection $\alpha-\Delta t C_{1}$, a diffusion term with coefficient $C_{2} F_{1}(\Delta t, h)$ and a reaction term $f(\bar{u})+\Delta t C_{3} F_{2}\left(f, f^{\prime}\right)$. Moreover if $C_{2} F_{1}(\Delta t, h) \neq 0$ the modified equation is of advection-diffusion-reaction type.

Let us now describe how the modified equation can give some insight on the qualitative behaviour of the numerical method. The construction of the modified PDE corresponding to the different splitting methods is a tedious but straightforward task. In what follows we present the methodology used in the construction of the modified equation associated with $I_{1}^{a} E_{1}^{r} I_{1}^{a}$. 
From Table 1, the method has the final form

$$
B_{h}^{2} u_{h}^{s+1}=u_{h}^{s}+\Delta t B_{h} f\left(B_{h}^{-1} u_{h}^{s}\right),
$$

where $B_{h}$ has only non null entries in the principal and lower diagonals which are equal to $1+r$ and $-r$ respectively. $B_{h}^{2}$ has only non null entries in the principal and the two lower diagonals which are equal to $(1+r)^{2},-2 r(1+r)$ and $r^{2}$ respectively; the matrix $B_{h}^{-1}$ has the following $i$-row

$$
\left(\frac{r^{i-1}}{(1+r)^{i}},(-1)^{i-2} \frac{r^{i-2}}{(1+r)^{i-1}}, \ldots, \frac{r}{(1+r)^{2}}, \frac{1}{1+r}, 0, \ldots, 0\right) .
$$

The general equation of (27) can be represented by

$$
\begin{aligned}
& r^{2} u_{j-2}^{s+1}+2 r(1-r) u_{j-1}^{s+1}+(1-r)^{2} u_{j}^{s+1} \\
& =u_{j}^{s}+\Delta t\left((1+r) f\left(\frac{1}{1+r} \sum_{\ell=0}^{j}\left(\frac{r}{1+r}\right)^{\ell}\right)\right. \\
& \left.\quad-r f\left(\frac{1}{1+r} \sum_{\ell=0}^{j-1}\left(\frac{r}{1+r}\right)^{\ell}\right)\right) .
\end{aligned}
$$

Assuming that the interpolation function $\bar{u}$ of $u_{h}^{s}$ is smooth enough we can use Taylor's formula concluding from (29) that

$$
\begin{aligned}
\frac{\partial \bar{u}}{\partial t}=-\alpha \frac{\partial \bar{u}}{\partial x} & -\frac{\Delta t}{2} \frac{\partial^{2} \bar{u}}{\partial t^{2}}-\frac{h \alpha}{2}(r-1) \frac{\partial^{2} \bar{u}}{\partial x^{2}}-\alpha \Delta t \frac{\partial^{2} \bar{u}}{\partial x \partial t} \\
& +\Delta t\left((1+r) f\left(\bar{u}_{j}\right)-r f\left(\bar{u}_{j}\right)\right)+\mathcal{O}\left(\Delta t^{2}, h^{2}\right)
\end{aligned}
$$

Eliminating $\frac{\partial^{2} \bar{u}}{\partial t^{2}}$ and $\frac{\partial^{2} \bar{u}}{\partial t \partial x}$ in (30) we conclude that $\bar{u}(x, t)$ is the exact solution of

$$
\frac{\partial \bar{u}}{\partial t}=-\alpha \frac{\partial \bar{u}}{\partial x}+\left(\frac{\alpha^{2} \Delta t}{4}+\frac{\alpha h}{2}\right) \frac{\partial^{2} \bar{u}}{\partial x^{2}}+f(\bar{u})-\frac{\Delta t}{2} f^{\prime}(\bar{u}) f(\bar{u})+\mathcal{O}\left(\Delta t^{2}, h^{2}\right) .
$$

This modified equation presents a parabolic character because it has a dissipative term and also exhibits a "wrong" reaction term represented by

$$
f(\bar{u})-\frac{\Delta t}{2} f^{\prime}(\bar{u}) f(\bar{u})+\mathcal{O}\left(\Delta t^{2}, h^{2}\right) .
$$

In the case of a production reaction $f(\bar{u})>0$ and for a loss reaction $f(\bar{u})<0$. As in production and loss reactions the reaction speed is a decreasing function of the concentration $\bar{u}$, we consider $f^{\prime}(\bar{u})<0$. The reaction term of the modified equation satisfies

$$
\left|f(\bar{u})-\frac{\Delta t}{2} f^{\prime}(\bar{u}) f(\bar{u})\right|>|f(\bar{u})|
$$

and, consequently, we can predict that the numerical solution given by $I_{1}^{a} E_{1}^{r} I_{1}^{a}$ is such that for a production reaction

$$
\bar{u}(x, t) \geq u(x, t), x \in[0,1], t \geq 0,
$$


and for a loss reaction

$$
\bar{u}(x, t) \leq u(x, t), x \in[0,1], t \geq 0 .
$$

In Figure 9 two numerical simulations show the effectiveness of our prediction both for loss and production reactions.
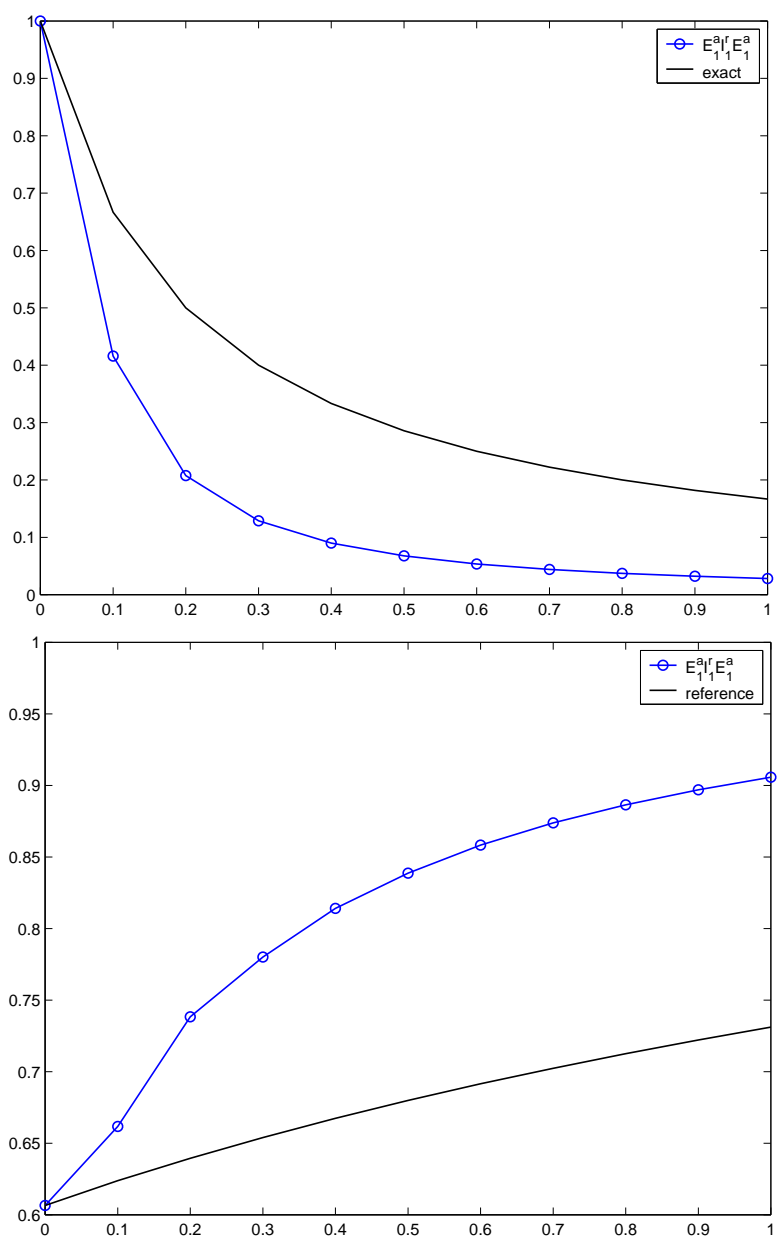

Fig. 9. Numerical results obtained for $t=10$ with $\Delta t=0.1$ and $h=0.1$ : (a) loss reaction $(\alpha=0.2, k=-1)$; (b) production reaction $(\alpha=1, k=1)$.

To correct the "wrong" reaction speed exhibited by $I_{1}^{a} E_{1}^{r} I_{1}^{a}$ we can use $I_{1}^{a} E_{2}^{r} I_{1}^{a}$ - a splitting method which corresponds to use an implicit upwind method for advection and a second order explicit Runge-Kutta method for reaction - as explained in what follows. From Table 1 we know that the global method takes the 
form

$$
B_{h}^{2} u_{h}^{s+1}=u_{h}^{s}+\Delta t(1-\gamma) B_{h} f\left(B_{h} u_{h}^{s}\right)+\Delta t \gamma B_{h} f\left(f\left(B_{h}^{-1} u_{h}^{s}\right)+\frac{\Delta t}{2 \gamma} f\left(B_{h}^{-1} u_{h}^{s}\right)\right),
$$

where $\gamma \in] 0,1]$.

Proceeding as before we establish the modified parabolic PDE associated with $(32)$ :

$$
\frac{\partial \bar{u}}{\partial t}=-\alpha \frac{\partial \bar{u}}{\partial x}+f(\bar{u})+\left(\frac{\alpha h}{2}+\frac{\alpha^{2} \Delta t}{4}\right) \frac{\partial^{2} \bar{u}}{\partial x^{2}}+\mathcal{O}\left(\Delta t^{2}, h^{2}\right) .
$$

We note that, while the reaction term is now first order accurate, a first order dissipation term is still present. This first order dissipation term arises from the integration of advection equations with an implicit method.

For method $I_{1}^{a} I_{1}^{r} I_{1}^{a}$ the modified PDE is

$$
\frac{\partial \bar{u}}{\partial t}=-\alpha \frac{\partial \bar{u}}{\partial x}+\left(\frac{\alpha h}{2}+\frac{\alpha^{2} \Delta t}{4}\right) \frac{\partial^{2} \bar{u}}{\partial x^{2}}+f(\bar{u})+\frac{\Delta t}{2} f^{\prime}(\bar{u}) f(\bar{u})+\mathcal{O}\left(\Delta t^{2}, h^{2}\right),
$$

which allows us to conclude that for a loss reaction,

$$
\bar{u}(x, t) \geq u(x, t) .
$$

The effects of wrong reaction speeds previously described for methods $I_{1}^{a} E_{1}^{r} I_{1}^{a}$, $I_{1}^{a} E_{2}^{r} I_{1}^{a}$ and $I_{1}^{a} I_{1}^{r} I_{1}^{a}$ can be observed in Figure 10.

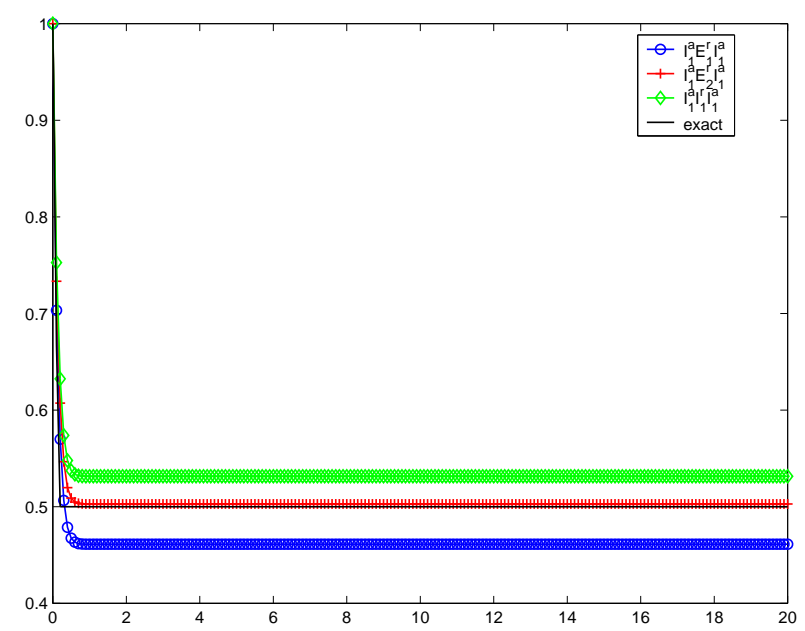

FIG. 10. Numerical results obtained with $I_{1}^{a} I_{1}^{r} I_{1}^{a}, I_{1}^{a} E_{1}^{r} I_{1}^{a}$ and $I_{1}^{a} E_{2}^{r} I_{1}^{a}$ for $\alpha=0.2, k=-1$ at $t=1$ in the spatial interval $[0,20]$ with $\Delta t=0.2, h=0.1$.

Methods $I_{1}^{a} I_{1}^{r} I_{1}^{a}, I_{1}^{a} E_{1}^{r} I_{1}^{a}$ and $I_{1}^{a} E_{2}^{r} I_{1}^{a}$ all present a dissipation coefficient $\frac{\alpha h}{2}+$ 
$\frac{\alpha^{2} \Delta t}{4}$. This is clearly observed in Figure 11.

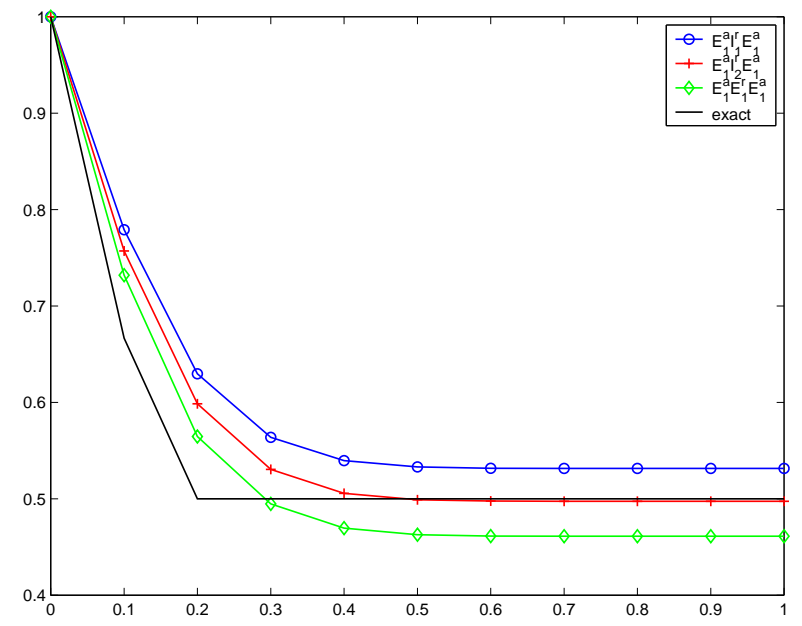

FIG. 11. A zoom of numerical results of Figure 9 in the spatial interval $[0,1]$.

To eliminate this spurious dissipation, we keep Runge-Kutta method in the reaction equation and increase the accuracy of the numerical method for advection equations using a second order implicit method for advection (Crank-Nicolson method)

$$
\frac{v_{j}^{s+1}-v_{j}^{s}}{\Delta t}+\frac{\alpha}{2}\left(\frac{v_{j+1}^{s+1}-v_{j-1}^{s+1}}{2 h}+\frac{v_{j+1}^{s}-v_{j-1}^{s}}{2 h}\right)=0,
$$

for $j=1, \ldots, n-1$, and

$$
\frac{v_{n}^{s+1}-v_{n}^{s}}{\Delta t}+\frac{\alpha}{2}\left(\frac{3 v_{n}^{s+1}-4 v_{n-1}^{s+1}+v_{n-2}^{s+1}}{2 h}+\frac{3 v_{n}^{s}-4 v_{n-1}^{s}+v_{n-2}^{s}}{2 h}\right)=0 .
$$

We represent this method by $I_{2}^{a} E_{2}^{r} I_{2}^{a}$. The final global method is represented by

$$
\begin{aligned}
& C_{h}^{(i)} C_{h}^{(e)-1} C_{h}^{(i)} u_{h}^{s+1} \\
& =C_{h}^{(e)} u_{h}^{s}+\Delta t\left((1-\gamma) C_{h}^{(i)} f\left(C_{h}^{(i)-1} C_{h}^{(e)} u_{h}^{s}\right)\right. \\
& \left.\quad+\gamma C_{h}^{(i)} f\left(f\left(C_{h}^{(i)-1} C_{h}^{(e)} u_{h}^{s}\right)+\frac{\Delta t}{2 \gamma} f\left(C_{h}^{(i)-1} C_{h}^{(e)} u_{h}^{s}\right)\right)\right) .
\end{aligned}
$$

The matrices $C_{h}^{(i)}$ and $C_{h}^{(e)}$ are defined as follows: $C_{h}^{(i)}$ is a tridiagonal matrix with entries $-\frac{\alpha \Delta t}{8 h}, 1, \frac{\alpha \Delta t}{8 h}$, excepted the $n^{\text {th }}$ line which is

$$
\left(0, \ldots, 0, \frac{\alpha \Delta t}{8 h},-\frac{\alpha \Delta t}{2 h}, 1+\frac{3 \alpha \Delta t}{8 h}\right)
$$


$C_{h}^{(e)}$ is a tridiagonal matrix with entries $\frac{\alpha \Delta t}{8 h}, 1,-\frac{\alpha \Delta t}{8 h}$ excepted the $n^{\text {th }}$ line which is

$$
\left(0, \ldots, 0,-\frac{\alpha \Delta t}{8 h}, \frac{\alpha \Delta t}{2 h}, 1-\frac{3 \alpha \Delta t}{8 h}\right) .
$$

Computing $C_{h}^{(i)} C_{h}^{(e)-1} C_{h}^{(i)}, C_{h}^{(i)-1} C_{h}^{(e)}$, we can establish after some tedious computations the modified PDE associated with $I_{2}^{a} E_{2}^{r} I_{2}^{a}$,

$$
\frac{\partial \bar{u}}{\partial t}=-\alpha \frac{\partial \bar{u}}{\partial x}+f(\bar{u})+\mathcal{O}\left(\Delta t^{2}, h^{2}\right)
$$

which has no first order diffusion terms, while keeping the correct reaction speed as $I_{1}^{a} E_{2}^{r} I_{1}^{a}$ (Figure 12).

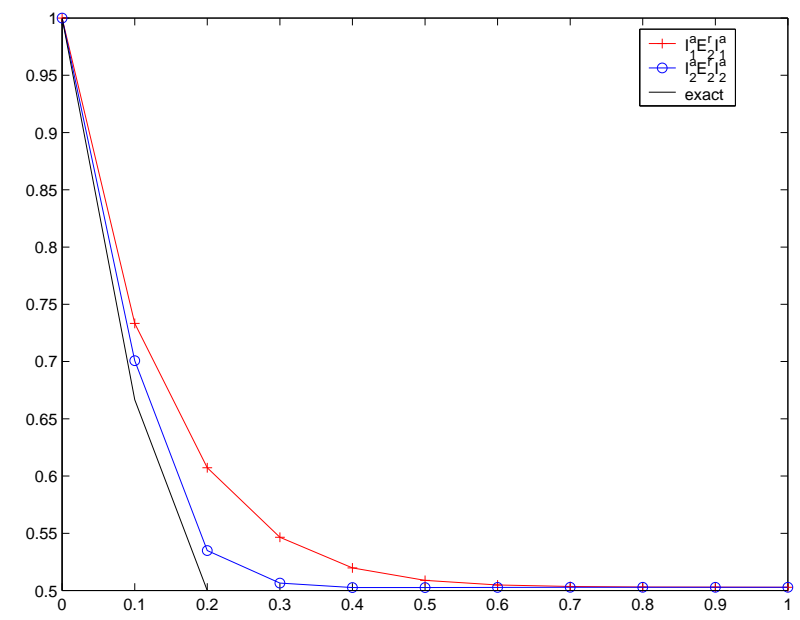

FIG. 12. Numerical results in the spatial interval $[0,1]$.

Let us now consider methods of type $E_{1}^{a} I_{1}^{r} E_{1}^{a}$ where (2), (4) are discretized with a first order explicit upwind method of type (12) and (3) is discretized with an implicit first order method (16). From Table 1 this global method can be written in the form

$$
u_{h}^{s+1}=A_{h}^{2} u_{h}^{s}+\Delta t A_{h} f\left(A_{h}^{-1} u_{h}^{s+1}\right) .
$$

In this case the modified equation approach lead us to

$$
\frac{\partial \bar{u}}{\partial t}=-\alpha \frac{\partial \bar{u}}{\partial x}+f(\bar{u})+\left(\frac{\alpha h}{2}-\frac{\alpha^{2} \Delta t}{4}\right) \frac{\partial^{2} \bar{u}}{\partial x^{2}}+\frac{\Delta t}{2} f^{\prime}(\bar{u}) f(\bar{u})+\mathcal{O}\left(\Delta t^{2}, h^{2}\right) .
$$

This numerical method introduces less numerical dissipation than the subclass of methods previously analysed but the reaction term now defined by

$$
f(\bar{u})+\frac{\Delta t}{2} f^{\prime}(\bar{u}) f(\bar{u})
$$




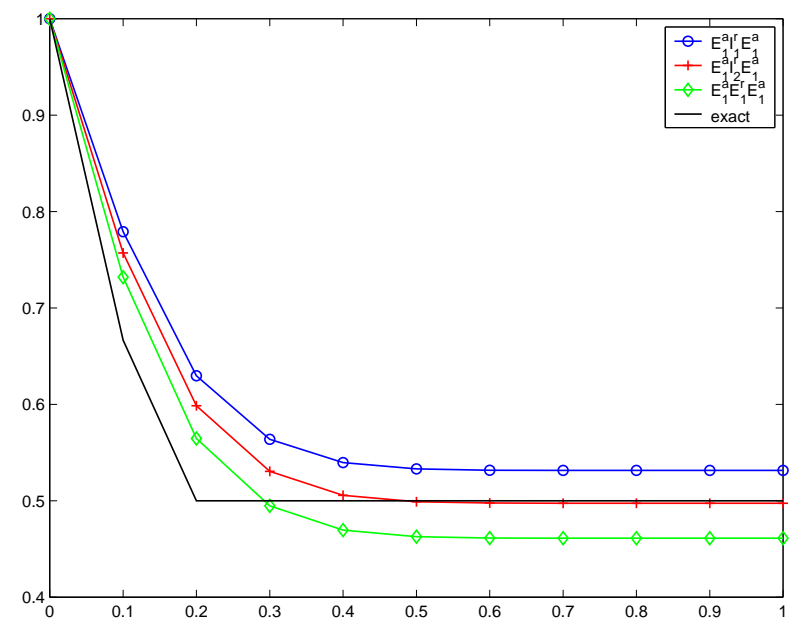

FIG. 13. Numerical results obtained with $E_{1}^{a} E_{1}^{r} E_{1}^{a}, E_{1}^{a} I_{1}^{r} E_{1}^{a}$ and $E_{1}^{a} I_{2}^{r} E_{1}^{a}$ for $\alpha=0.2, k=-1$ at $t=1$ in the spatial interval $[0,1]$ with $\Delta t=0.2, h=0.1$.

lead us to the time step restriction (22) already obtained in the stability study of Section 3 and which guarantees that there is no inversion in the kinetics. In fact, if $(22)$ is satisfied the numerical reaction term is such that

$$
\left|f(\bar{u})+\frac{\Delta t}{2} f^{\prime}(\bar{u}) f(\bar{u})\right| \leq|f(\bar{u})|,
$$

and we can predict that the numerical solution given by $E_{1}^{a} I_{1}^{r} E_{1}^{a}$ is such that, for a production reaction,

$$
\bar{u}(x, t) \leq u(x, t), x \in[0,1], t \geq 0,
$$

and for a loss reaction

$$
\bar{u}(x, t) \geq u(x, t), \quad x \in[0,1], t \geq 0 .
$$

In Figure 13 the prediction is confirmed for a loss reaction. To correct the "wrong" reaction term while preserving the dissipative term we construct the numerical method $E_{1}^{a} I_{2}^{r} E_{1}^{a}$ that corresponds to integrate (2) and (4) with the explicit first order method (12) and equation (3) with the implicit second order trapezoidal method (17). From Table 1 the global method is then defined by

$$
u_{h}^{s+1}=A_{h}^{2} u_{h}^{s}+\frac{\Delta t}{2}\left(A_{h} f\left(A_{h}^{-1} u_{h}^{s+1}\right)+A_{h} f\left(A_{h} u_{h}^{s}\right)\right) .
$$

Assuming enough smoothness of $f$, the modified equation approach lead us to

$$
\frac{\partial \bar{u}}{\partial t}=-\alpha \frac{\partial \bar{u}}{\partial x}+f(\bar{u})+\left(\frac{\alpha h}{2}-\frac{\alpha^{2} \Delta t}{4}\right) \frac{\partial^{2} \bar{u}}{\partial x^{2}}+\mathcal{O}\left(\Delta t^{2}, h^{2}\right),
$$


which presents the same first order error in the dissipation as before. The plot in Figure 13 shows that the reaction term has been corrected with $E_{1}^{a} I_{2}^{r} E_{1}^{a}$.

\begin{tabular}{|l||l|}
\hline Method & Modified PDE \\
\hline \hline NSE & $\frac{\partial \bar{u}}{\partial t}=-\alpha \frac{\partial \bar{u}}{\partial x}+f(\bar{u})+\left(\frac{\alpha h}{2}-\frac{\alpha^{2} \Delta t}{2}\right) \frac{\partial^{2} \bar{u}}{\partial x^{2}}+\alpha \Delta t f^{\prime}(\bar{u}) \frac{\partial \bar{u}}{\partial x}-\frac{\Delta t}{2} f^{\prime}(\bar{u}) f(\bar{u})+\mathcal{O}\left(\Delta t^{2}, h^{2}\right)$ \\
$E_{1}^{a} E_{1}^{r} E_{1}^{a}$ & $\frac{\partial \bar{u}}{\partial t}=-\alpha \frac{\partial \bar{u}}{\partial x}+f(\bar{u})+\left(\frac{\alpha h}{2}-\frac{\alpha^{2} \Delta t}{4}\right) \frac{\partial^{2} \bar{u}}{\partial x^{2}}-\frac{\Delta t}{2} f^{\prime}(\bar{u}) f(\bar{u})+\mathcal{O}\left(\Delta t^{2}, h^{2}\right)$ \\
$E_{1}^{a} I_{1}^{r} E_{1}^{a}$ & $\frac{\partial \bar{u}}{\partial t}=-\alpha \frac{\partial \bar{u}}{\partial x}+f(\bar{u})+\left(\frac{\alpha h}{2}-\frac{\alpha^{2} \Delta t}{4}\right) \frac{\partial^{2} \bar{u}}{\partial x^{2}}+\frac{\Delta t}{2} f^{\prime}(\bar{u}) f(\bar{u})+\mathcal{O}\left(\Delta t^{2}, h^{2}\right)$ \\
$E_{1}^{a} I_{2}^{r} E_{1}^{a}$ & $\frac{\partial \bar{u}}{\partial t}=-\alpha \frac{\partial \bar{u}}{\partial x}+f(\bar{u})+\left(\frac{\alpha h}{2}-\frac{\alpha^{2} \Delta t}{4}\right) \frac{\partial^{2} \bar{u}}{\partial x^{2}}+\mathcal{O}\left(\Delta t^{2}, h^{2}\right)$ \\
NSI & $\frac{\partial \bar{u}}{\partial t}=-\alpha \frac{\partial \bar{u}}{\partial x}+f(\bar{u})+\left(\frac{\alpha h}{2}+\frac{\alpha^{2} \Delta t}{2}\right) \frac{\partial^{2} \bar{u}}{\partial x^{2}}-\alpha \Delta t f^{\prime}(\bar{u}) \frac{\partial \bar{u}}{\partial x}+\frac{\Delta t}{2} f^{\prime}(\bar{u}) f(\bar{u})+\mathcal{O}\left(\Delta t^{2}, h^{2}\right)$ \\
$I_{1}^{a} I_{1}^{r} I_{1}^{a}$ & $\frac{\partial \bar{u}}{\partial t}=-\alpha \frac{\partial \bar{u}}{\partial x}+f(\bar{u})+\left(\frac{\alpha h}{2}+\frac{\alpha^{2} \Delta t}{4}\right) \frac{\partial^{2} \bar{u}}{x^{2}}+\frac{\Delta t}{2} f^{\prime}(\bar{u}) f(\bar{u})+\mathcal{O}\left(\Delta t^{2}, h^{2}\right)$ \\
$I_{1}^{a} E_{1}^{r} I_{1}^{a}$ & $\frac{\partial \bar{u}}{\partial t}=-\alpha \frac{\partial \bar{u}}{\partial x}+f(\bar{u})+\left(\frac{\alpha h}{2}+\frac{\alpha^{2} \Delta t}{4}\right) \frac{\partial^{2} \bar{u}}{\partial x^{2}}-\frac{\Delta t}{2} f^{\prime}(\bar{u}) f(\bar{u})+\mathcal{O}\left(\Delta t^{2}, h^{2}\right)$ \\
$I_{1}^{a} E_{2}^{r} I_{1}^{a}$ & $\frac{\partial \bar{u}}{\partial t}=-\alpha \frac{\partial \bar{u}}{\partial x}+f(\bar{u})+\left(\frac{\alpha h}{2}+\frac{\alpha^{2} \Delta t}{4}\right) \frac{\partial^{2} \bar{u}}{\partial x^{2}}+\mathcal{O}\left(\Delta t^{2}, h^{2}\right)$ \\
$I_{2}^{a} E_{2}^{r} I_{2}^{a}$ & $\frac{\partial \bar{u}}{\partial t}=-\alpha \frac{\partial \bar{u}}{\partial x}+f(\bar{u})+\mathcal{O}\left(\Delta t^{2}, h^{2}\right)$ \\
\hline
\end{tabular}

TABle 2. Modified PDE's.

In Table 2 we present the modified equations corresponding to the different methods of the class presented in Section 2. We also include the modified equations associated with methods NSE and NSI.

As far as advection and reaction terms of numerical flows are concerned, we note that they are both increasing functions of $\Delta t$ (or decreasing functions of $\Delta t$ ) in the case of NSE (or NSI). This means that the first order errors in advection and reaction can be cancelled and no prediction is allowed from the modified equation approach.

\section{Conclusions}

In this paper several splitting methods were studied considering three aspects: stability, accuracy and qualitative behaviour. The influence of explicitness, implicitness and order in the stability of the global method was analyzed and stability estimates were established. These estimates allow us to select, a priori, a splitting method with specified stability properties. This selection takes into account the stiffness of the problem: for stiff problems, reaction should be integrated with implicit methods, while for non-stiff or mildly stiff problems explicit methods can be used.

The influence of explicitness, implicitness and order in the qualitative behaviour of splitting methods was also object of study. Using the modified equation approach we are able to predict the qualitative behaviour of splitting methods and also to correct erratic numerical flow obtained using some splitting methods. 


\section{References}

[1] A. Araújo, J. A. Ferreira, P. Oliveira, F. Patrício and P. Rosa, The use of splitting methods in the numerical simulation of reacting flows, to appear in Computing and Visualization in Science.

[2] U. M. Ascher, S. J. RuUth And B. Wetton, Implicit-explicit methods for time-dependent partial differential equations, SIAM J. Numer. Anal. 32 (1995), 797-823.

[3] J. Douglas, Aternating direction methods for three space variables, Numer. Math. 4 (1962), 41-63.

[4] J. Douglas and J. E. GunN, A general formulation of alternating direction methods, Numer. Math. 6 (1996), 428-453.

[5] W. H. Hundsdorfer, Trapezoidal and midpoint splitting for initial-boundary value problems, Math. Comp. 67 (1998), 1047-1062.

[6] W. H. Hundsdorfer, A note on stability of the Douglas splitting method, Math. Comp. 67 (1998), 183-190.

[7] W. H. HUNDSDORFER, Splitting techniques for advection - diffusion - reaction equations, in: Proceedings of the Workshop Modelling and simulation in chemical engineering, Coimbra, June 30 - July 4, 2003, A. E. Rodrigues, P. de Oliveira, J. A. Castro, J. A. Ferreira and M. C. Colmbra (eds.).

[8] R. J. Leveque, Numerical Methods for Conservation Laws, Birkhäuser-Verlag, Basel, 1990.

[9] L. A. Monthé, A study of splitting scheme for hyperbolic conservation laws with source terms, J. Comp. Appl. Math. 137 (2001), 1-12.

[10] E. S. Oran and J. P. Boris, Numerical Simulation of Reactive Flows, Elsevier, 1987.

[11] E. J. Spee, J. G. Verwer, P. M. de Zeuw, J. G. Blom and W. H. Hundsdorfer, A numerical study for global atmospheric transport-chemistry problems, Mathematics and Computers in Simulation 48 (1998), 177-204.

A. Araújo, J. A. Ferreira and P. de Oliveira

Departamento de Matemática

Universidade de Coimbra

Portugal

(accepted: September 2, 2004)

\section{To access this journal online: (40) http://www.birkhauser.ch}

